DrAFt VERSion June 22, 2021

Preprint typeset using $\mathrm{IAT}_{\mathrm{E}} \mathrm{X}$ style emulateapj v. 5/2/11

\title{
THE EFFECT OF RADIAL MIGRATION ON GALACTIC DISKS
}

\author{
Carlos Vera-Ciro $^{1}$, Elena D’Onghia ${ }^{1,4}$, Julio Navarro ${ }^{2,5}$, Mario Abadi ${ }^{3}$ \\ Draft version June 22, 2021
}

\begin{abstract}
We study the radial migration of stars driven by recurring multi-arm spiral features in an exponential disk embedded in a dark matter halo. The spiral perturbations redistribute angular momentum within the disk and lead to substantial radial displacements of individual stars, in a manner that largely preserves the circularity of their orbits and that results, after 5 Gyr $(\sim 40$ full rotations at the disk scalelength), in little radial heating and no appreciable changes to the vertical or radial structure of the disk. Our results clarify a number of issues related to the spatial distribution and kinematics of migrators. In particular, we find that migrators are a heavily biased subset of stars with preferentially low vertical velocity dispersions. This "provenance bias" for migrators is not surprising in hindsight, for stars with small vertical excursions spend more time near the disk plane and thus respond more readily to non-axisymmetric perturbations. We also find that the vertical velocity dispersion of outward migrators always decreases, whereas the opposite holds for inward migrators. To first order, newly arrived migrators simply replace stars that have migrated off to other radii, thus inheriting the vertical bias of the latter. Extreme migrators might therefore be recognized, if present, by the unexpectedly small amplitude of their vertical excursions. Our results show that migration, understood as changes in angular momentum that preserve circularity, can affect strongly the thin disk, but cast doubts on models that envision the Galactic thick disk as a relic of radial migration.

Subject headings: galaxies: kinematics and dynamics - Galaxy: disk - Galaxy: evolution - stars: kinematics and dynamics
\end{abstract}

\section{INTRODUCTION}

Cold, thin stellar disks are dynamically fragile entities that react strongly to changes in the gravitational potential, such as those that result from changes in the mass of the galaxy itself, from external perturbations such as the accretion of a satellite or the tidal effects of substructure, or from internal processes such as bar instability or the development of spiral structure. In the absence of dissipation, these perturbations generally heat the disk, moving stars away from their birth radii at the expense of decreasing the circularity of their orbits and increasing the amplitude of their vertical oscillations. Stars on nearly circular orbits must have therefore been born at about the same radius where they are found today.

One difficulty with this simple scenario arises in our Galaxy from the weak correlation between age and metallicity for thin disk stars in the solar neighborhood (Edvardsson et al. 1993), which is essentially flat for ages between 0 and 8 Gyr (see, e.g., Bergemann et al. 2014, and references therein). Although this may be taken to suggest a simple (but somewhat contrived) scenario where the average metallicity of the interstellar medium (ISM) at the solar circle has not changed since $z \sim 1$, the sizable scatter in the correlation would also require the ISM to have been rather in-homogeneous in the past, a possibility that seems at face value unattractive (see Feltzing

\footnotetext{
${ }^{1}$ Department of Astronomy, University of Wisconsin, 2535 Sterling Hall, 475 N. Charter Street, Madison, WI 53076, USA. e-mail:ciro@astro.wisc.edu

${ }^{2}$ Department of Physics and Astronomy, University of Victoria, Victoria, BC, V8P 5C2, Canada

3 Observatorio Astronómico, Universidad Nacional de Córdoba, Córdoba X5000BGR, Argentina

${ }^{4}$ Alfred P. Sloan Fellow

${ }^{5}$ Senior CIfAR Fellow
}

\& Chiba 2013, for a recent review).

An alternative view holds that thin disk stars do not necessarily remain close to their birth radii (Wielen et al. 1996). If true, then long-lived stars in the solar neighborhood might actually sample the chemical properties of the Galaxy over a wide range of radii at the time of their formation, possibly blurring any correlation between the age and metallicity of stars selected in local samples. This scenario gained support after Sellwood and Binney, in an influential paper, identified a mechanism that allows stars to migrate in radius whilst remaining on nearly circular orbits (Sellwood \& Binney 2002). The mechanism relies on angular momentum exchanges between stars near corotation of transient spiral patterns, and allows stars to switch radii without substantially heating the disk. Given that spiral patterns seem ubiquitous in disk galaxies, this mechanism must operate to some degree in most galaxy disks.

Since its inception, the corotation resonance (CR) mechanism of Sellwood \& Binney (2002) has been the subject of a number of studies in the literature. These studies have generally confirmed the validity of the arguments laid out in their original paper and extended their application to multiple, interacting spiral patterns (Minchev \& Famaey 2010; Brunetti et al. 2011), but have also raised a number of questions about the initial and final properties of migrating stars and about the consequences of radial migration for the vertical structure of the disk. It is unclear, for example, what biases, if any, might affect the kinematics or spatial distribution of migrators, what strategies might be used to identify migrators observationally, or whether radial migration plays a role in the origin of the thick disk of the Galaxy.

The latter issue, in particular, has been debated vig- 


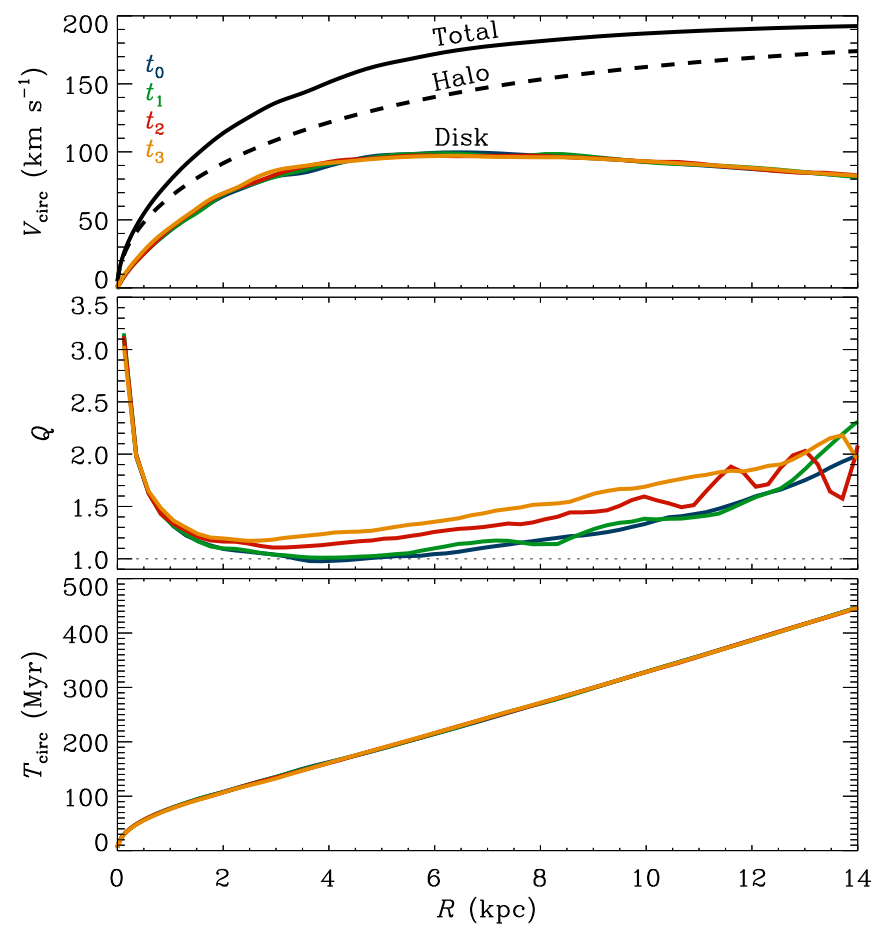

Figure 1. Properties of the galaxy model. Top: circular velocity, $V_{\text {circ }}^{2}=R \partial \Phi / \partial R$, profile for the spiral simulation, at various times. No substantial evolution is observed in the overall mass distribution, even after 5 Gyr of evolution. The contribution of the disk at various times is shown by the colored curves; that of the (static) dark halo is shown by the dashed line; the total is indicated by the black solid line. Middle: Toomre's $Q$ parameter profile of the spiral disk at different times. Bottom: circular orbit period, $T_{\text {circ }}=2 \pi R / V_{\text {circ }}$, as a function of radius at different times.

orously, especially since Schönrich \& Binney (2009) proposed that the chemical differentiation between thin and thick disks could be explained solely by radial migration, which might have brought stars with high vertical kinetic energy from the inner Galaxy out to the solar neighborhood. Minchev et al. (2012b), on the other hand, have argued that migration does little for disk thickening because outward migrators should cool vertically as they move out, a result that has been further debated by Solway et al. (2012) and Roškar et al. (2013).

This unsettled state of affairs might be traced, in part, to the disparate numerical contexts used to study the effects of migration. Although most published work has relied on direct numerical simulation, some studies are based on the response of equilibrium disk models to transient spiral modes (Sellwood \& Binney 2002; Grand et al. 2012; Solway et al. 2012; Sellwood \& Carlberg 2014) or to the tidal perturbation of a massive satellite (Kazantzidis et al. 2008; Villalobos \& Helmi 2008; Bird et al. 2012), whereas others have focused on hydrodynamical simulations of disks of gas and stars that evolve in isolation (Minchev et al. 2011, 2012b) or that result from the dissipative collapse of a rotating sphere of gas in the gravitational potential of a dark matter halo (Roškar et al. 2008, 2012, 2013; Loebman et al. 2011).

The first approach benefits from the controlled nature of the initial disk, which allows for the identification of numerical artifact, but suffers from the arbitrariness in- volved in choosing the orbit and mass of the perturbers, or from differences in the origin of the spiral perturbations, which either develop freely or result from the forcing of particular modes with predetermined amplitude and duration. The second approach allows the disk to evolve more freely, but its analysis is obscured by the complexities that arise from the continuously evolving disk potential and from the presence of rapidly evolving multiple modes and instabilities. Furthermore, the numerical resolution of hydrodynamical simulations is inevitably much lower than that attainable with $N$-body models, making it much harder to discern and isolate the effects of noise associated with the finite number of particles used.

Finally, there are also semantic issues, since some authors use the term "radial migration" as a catch-all phrase to denote any radial displacement of a star, regardless of its cause or effect on its orbit, whereas others reserve the term "migration" for changes in the guiding center radius (i.e., the orbital angular momentum) of orbits that remain nearly circular through the process. We shall adopt the latter meaning in this contribution.

We address some of these issues here using numerical simulations that evolve a kinematically cold selfgravitating disk of stars in the fixed potential of a cosmologically motivated dark matter halo. Spiral patterns are seeded by perturbations modeled on the gravitational influence of molecular clouds and persist almost unchanged for over $5 \mathrm{Gyr}$, the duration of the simulation (more than 40 full rotations at the exponential disk scalelength). Our simulations differ from earlier work mainly on numerical resolution: the disk is made up of $10^{8}$ particles, an improvement of nearly two orders of magnitude over earlier work. The large number of particles allows us to study the orbital evolution of individual stars without having to worry about the discreteness noise associated with the particle representation of the disk.

Our simulations also differ from earlier attempts because the disk is not subject to the rapid development of instabilities that may change its radial or vertical structure; a control run without perturbers shows that the disk does not develop strong non-axisymmetric features over several rotation periods. Finally, our simulations also stand out because the spiral features seeded by the "molecular clouds" do not heat appreciably the disk and persist for over 5 Gyr. Both the vertical and azimuthally averaged structure of the disk remain basically unchanged throughout.

The plan for this paper is as follows. We describe the numerical simulations used in this work in Section 2, present our main results in Section 3, and conclude with a brief summary in Section 4.

\section{NUMERICAL METHODS}

\subsection{The Galaxy Model}

We use here $N$-body simulations of the same galaxy model presented by D'Onghia et al. (2013) . For the sake of completeness we give here a brief description of the simulations and refer the interested reader to the original reference for further details. The models evolve a self-gravitating disk of particles embedded in a rigid halo potential described by a Hernquist profile (Hernquist 1990$)$ with total mass $M_{\text {halo }}=9.5 \times 10^{11} \mathrm{M}_{\odot}$ and 


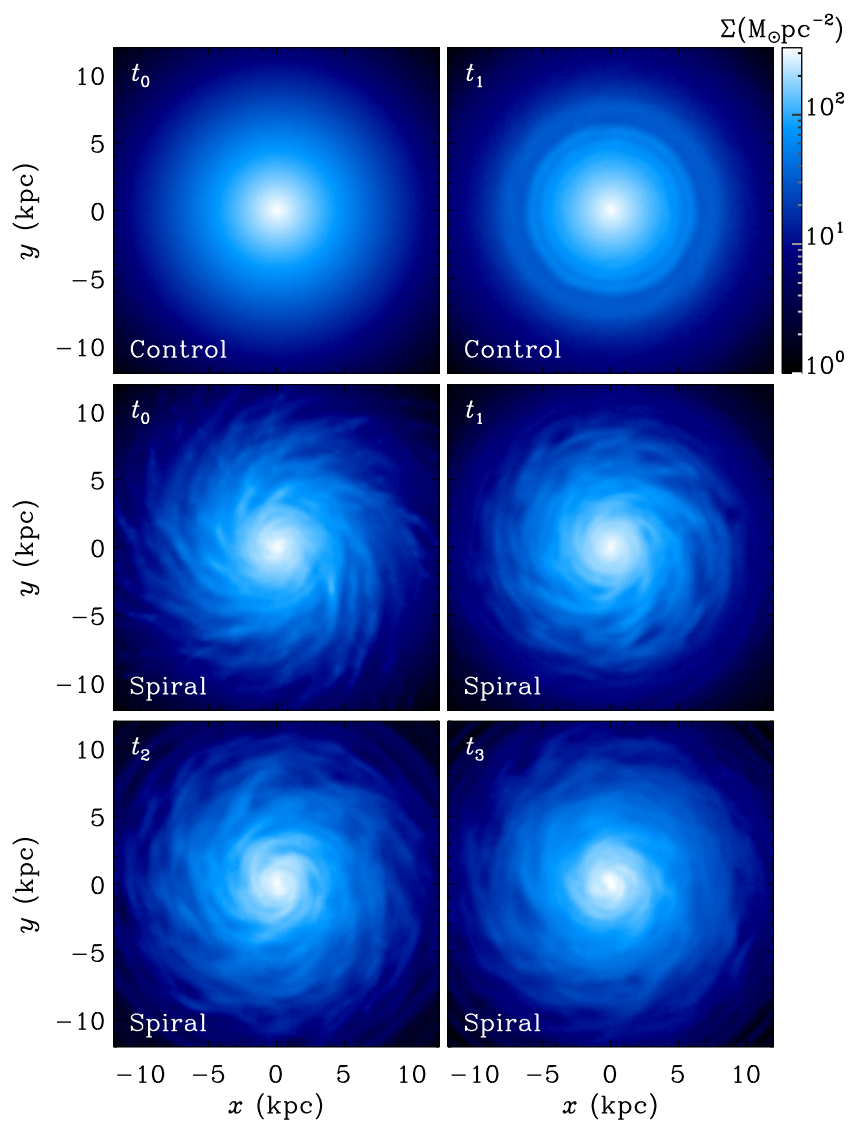

Figure 2. Face-on projected stellar surface density of the simulations at different times, as labeled. The top row shows the control simulation; the others correspond to the "spiral" disk. For the spiral disk, $t_{0}$ corresponds to the time just after the molecular-cloud perturbers are removed. Note that at that time the spiral arms are already in place. The times shown correspond to times measured after $t_{0}: t_{1}=831 \mathrm{Myr}, t_{2}=3032 \mathrm{Myr}$, and $t_{3}=5477 \mathrm{Myr}$.

scale length $r_{\text {halo }}=29.8 \mathrm{kpc}$.

The disk is a $10^{8}$ particle realization of an exponential disk of constant scaleheight:

$$
\begin{aligned}
\rho(R, z) & =\Sigma(R) \zeta(z) \\
& =\frac{M_{\text {disk }}}{4 \pi R_{\text {disk }}^{2} z_{0}} \exp \left(-\frac{R}{R_{\text {disk }}}\right) \operatorname{sech}^{2}\left(\frac{z}{z_{0}}\right),
\end{aligned}
$$

whose positions and velocities were generated using a modified version of the method presented by Hernquist (1993), as described by Springel et al. (2005). We adopt a radial scalelength $R_{\text {disk }}=3.13 \mathrm{kpc}$, scaleheight $z_{0}=$ $0.1 R_{\text {disk }}=0.313 \mathrm{kpc}$ and total disk mass $M_{\text {disk }}=1.81 \times$ $10^{10} \mathrm{M}_{\odot}$.

With these choices, the disk is submaximal, and makes up about $27 \%$ of the total mass enclosed within two radial scalelengths. A particle in a circular orbit completes a full rotation at $R_{\text {disk }}$ in $136 \mathrm{Myr}$. We show in Figure 1 the circular velocity profile of the galaxy model (top panel), and the circular orbit timescale, $T_{\text {circ }}=2 \pi R / V_{\text {circ }}(R)$, in Myr (bottom panel). The middle panel shows the Toomre stability parameter $Q=\sigma_{R} \kappa /(3.36 G \Sigma)$, chosen to ensure the disk is marginally stable at all radii. (Here $\kappa$ is the epicycle frequency and $\sigma_{R}$ the velocity dispersion

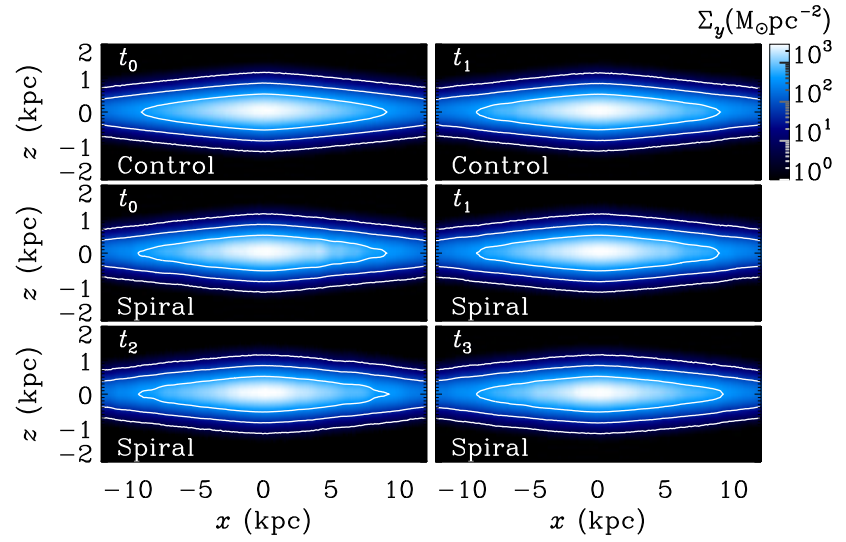

Figure 3. Same as Figure 2 but for the edge-on projections of the disk.

in radial motions.)

\subsection{The Control Simulation}

We have evolved the galaxy model described in the previous section for from $t_{0}=0 \mathrm{Myr}$ to $t_{1}=831 \mathrm{Myr}$, corresponding to six circular orbit timescales at $R_{\text {disk }}$, or three full rotations at $R=8 \mathrm{kpc}$. The system was evolved using an updated version of GADGET (see Springel 2005), using a Plummer-equivalent softening length for the stellar particles of $5 \mathrm{pc}$ and default integration parameters.

After 831 Myr the "control" disk has evolved little from its initial configuration, as may be seen in the top two panels of Figure 2, and has developed no obvious nonaxisymmetric features. It does develop, however, "ringlike" features that travel like radial waves through the disk, and which are easily appreciate in the top right panel of Figure 2.

These waves do not seem to be caused by discreteness effects, since they appear spontaneously and are coherent over the whole disk. The radial waves seem to travel back and forth through the disk, without causing any discernable change in the radial distribution of disk particles. They do not perturb appreciably the vertical structure of the disk, as shown in the top two panels of Figure 3, nor change the surface density profile of the disk, as shown in the left panel of Figure 4.

The left panels of Figure 5 show that the radial waves have little effect on the kinematics of disk particles: there is little evolution in the velocity dispersion profiles of the disk over the 831 Myr that the disk runs unperturbed. We conclude that our galaxy disk model is not subject to the strong non-axisymmetric instabilities that have plagued $N$-body studies of disk galaxies over the years. Although our disks are fully self-gravitating, it appears as if the number of particles used is sufficient to suppress discreteness noise and to prevent, for several rotation periods, the development of strong non-axisymmetric features.

\subsection{The "Spiral" Simulation}

As shown by D'Onghia et al. (2013), non-axisymmetric features do develop when the disk is perturbed in a manner consistent with the presence of $\sim 1000$ "molecular clouds". We refer the reader to D'Onghia et al. (2013) for details on the numerical implementation but, in brief, the 


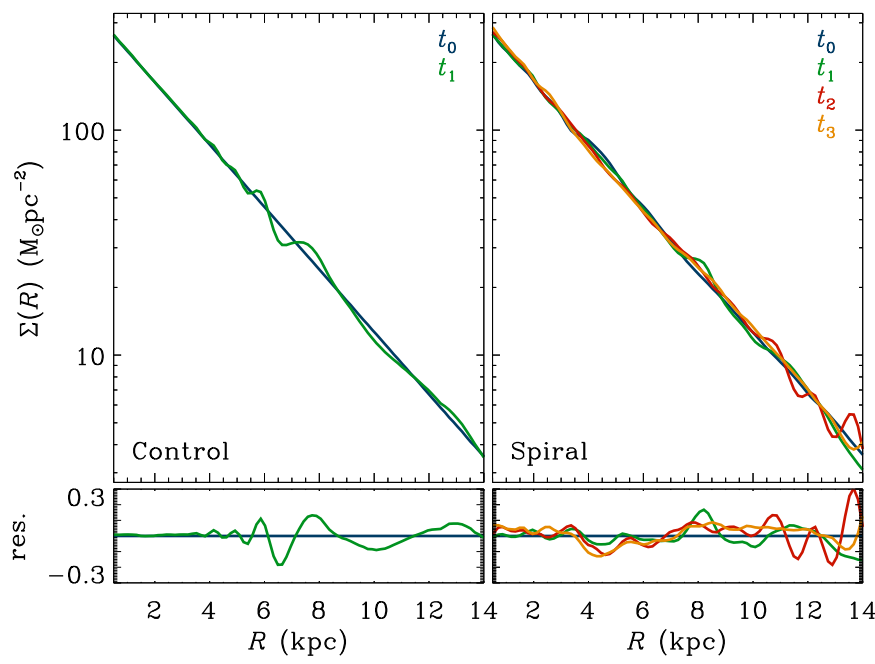

Figure 4. Surface density profile for the control simulation (left) and the spiral simulation (right). Colors correspond to different times, as labeled. The residuals for the density are calculated as $\ln \Sigma(R, t) / \Sigma\left(R, t_{0}\right)$ and are shown in the bottom panels. With the exception of radial density waves probably associated with small inaccuracies in the generation of initial conditions, no systematic change in the exponential shape of these profiles is observed.

perturbations are modeled by the addition of softened, $9.5 \times 10^{5} M_{\odot}$ particles constrained to travel in circular orbits and scattered about the disk. The perturbation is transient, and the molecular-cloud perturbers are removed 245 Myr after their inclusion.

The disk reacts strongly to these perturbations, and develops a long-lived multi-arm spiral pattern, as shown in the bottom four panels of Figure 2. In this sequence, $t_{0}$ shows the disk just after the perturbers are removed; $t_{1}$ corresponds, as in the control simulation, to $831 \mathrm{Myr}$ later; whereas $t_{2}$ and $t_{3}$ denote later stages of the evolution, 3032 Myr and 5477 Myr after the removal of the perturbers, respectively.

Visual inspection of Figure 2 shows that the multi-arm spirals seeded by the molecular cloud perturbers are long lived and that they change little in nature over $\sim 5 \mathrm{Gyr}$ of evolution. Despite their longevity, the spiral patterns seem to have little effect on the vertical structure of the disk, as shown by the bottom four panels of Figure 3 . The radial structure of the disk also remains almost unchanged, as indicated by the azimuthally averaged surface density profiles at $t=t_{0}, t_{1}, t_{2}$, and $t_{3}$ shown in the right-hand panel of Figure 4 .

Only the in-plane kinematics of the disk seems to be noticeably affected, as shown by the slight, but monotonic, heating in the radial and azimuthal directions seen in the right-hand panels of Figure 5. The vertical structure of the disk, however, is unaffected throughout: the vertical velocity dispersion profile, $\sigma_{z}(R)$, at $t_{3}$ is indistinguishable from that at $t=t_{0}$ (see bottom right panel of Figure 5).

\subsection{Azimuthal Structure}

We examine the azimuthal structure of the disks in Figure 6, where we show the fluctuations in the surface density and in the gravitational potential at various times as a function of the azimuthal angle $\theta$ and of the cylin-

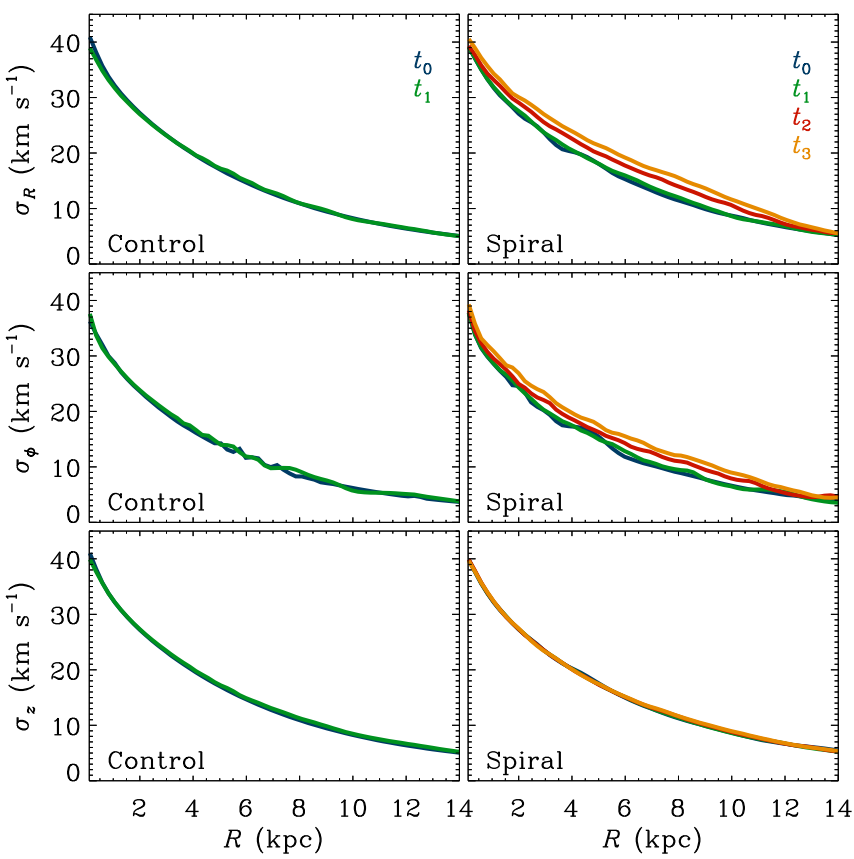

Figure 5. Radial (top), azimuthal (middle) and vertical (bottom) velocity dispersion profiles as a function of radius, for the control simulation (left) and the spiral simulation (right). Different colors correspond to different times, as labeled. The vertical component $\sigma_{z}$ is remarkably constant even after $\sim 5$ Gyr of evolution, with changes smaller than $\sim 3 \%$. On the other hand, the in-plane components increase slightly but steadily a function of time. The most affected is $\sigma_{R}$, which increases by $\sim 30 \%$ at $R=9 \mathrm{kpc}$ at the final time.

drical radius, $R$.

The leftmost panels of Figure 6 shows that a barely discernible multi-arm spiral pattern develops in the control simulation after 831 Myr of evolution. Given its small amplitude (the rms fluctuations in the surface density are just $5 \%$ at $R=5 \mathrm{kpc}$ ) this pattern is only evident in the residuals and, as we will see below, leads to basically no secular evolution in the disk over the $831 \mathrm{Myr}$ it was evolved for. This confirms our earlier statement that the model galaxy is free from the violent instabilities or rapidly-growing non-axisymmetric disturbances characteristic of models where either the galaxy is initially out of equilibrium, or where discreteness noise seeds perturbations that transform the galaxy over a short period of time.

The multi-arm spiral structure of the "spiral" simulation stands out clearly in Figure 6, with interarm surface density fluctuations of roughly $\pm 30 \%$. The actual gravitational potential fluctuations are much smaller, however, due to the presence of the dark matter halo. The spiral structure in the "spiral" simulation remains roughly constant from $t_{0}$ to $t_{3}$, and is characterized by a radially increasing number of arms that bifurcate outward: at $R=2 \mathrm{kpc}$, near the center, only about 4 arms are clearly defined, whereas more than 8 arms can be counted $10 \mathrm{kpc}$ away from the center. We ascribe the very slight evolution in the spiral structure from $t_{0}$ to $t_{3}$ to the weak, but monotonic, radial heating of the disk seen in the increase of the radial velocity dispersion shown in Figure 5. We emphasize again that the 

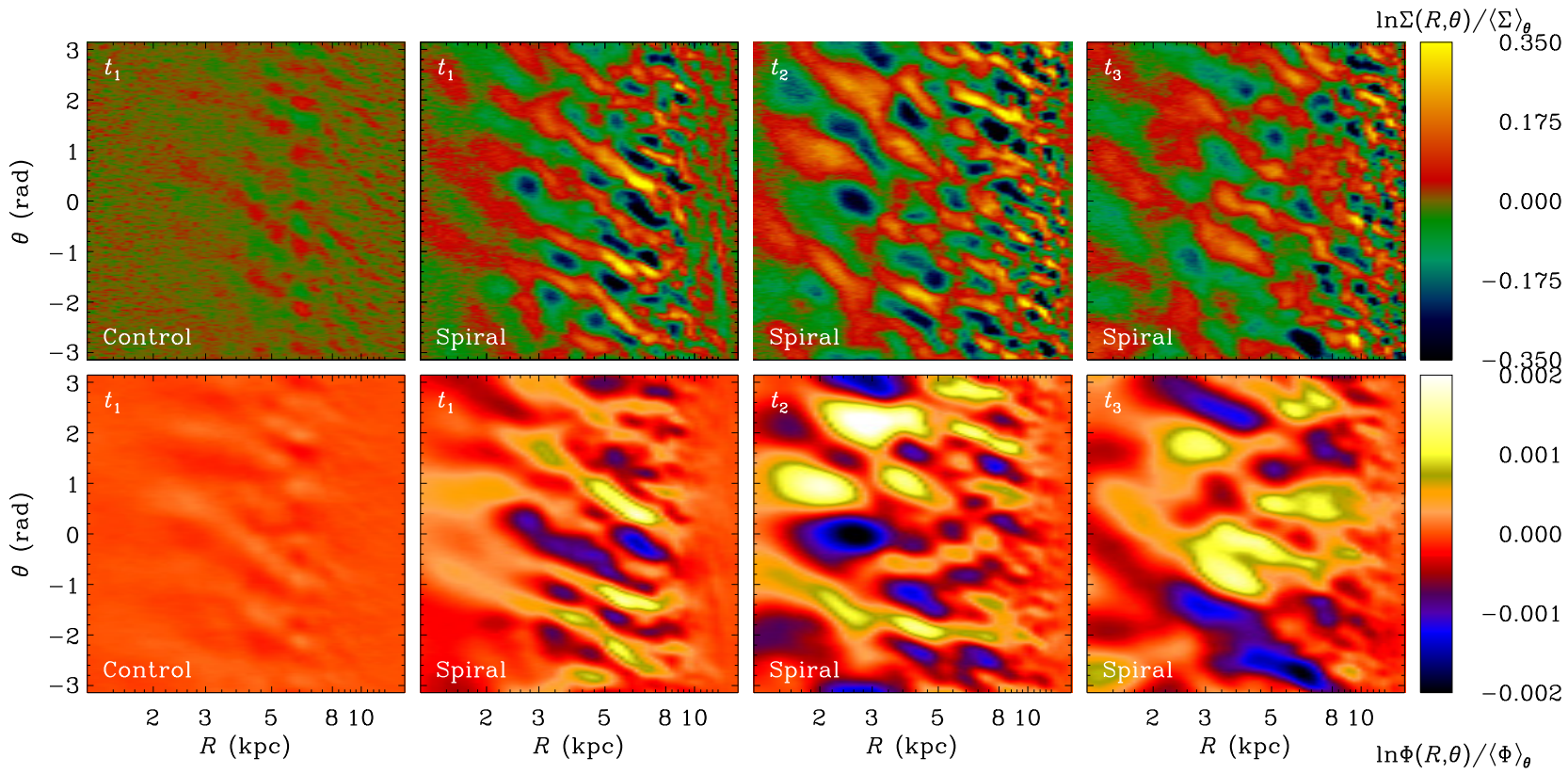

Figure 6. Fluctuations in the surface density (top row) and the gravitational potential at various times for the control simulation (leftmost column) and for the spiral simulation. The residuals are computed relative to the azimuthally averaged value at given radius as $\ln \Sigma(R, \theta) /\langle\Sigma\rangle_{\theta}$ and $\ln \Phi(R, \theta) /\langle\Phi\rangle_{\theta}$. The interarm fluctuations due to the spiral patterns is roughly $\sim 30 \%$ in the spiral simulation, but only $\sim 0.1 \%$ in the potential.

overall heating is quite modest; in 5 Gyr of evolution the radial velocity dispersion at $R=5 \mathrm{kpc}$ increases from $18 \mathrm{~km} \mathrm{~s}^{-1}$ to just over $21 \mathrm{~km} \mathrm{~s}^{-1}$. The increase is even smaller at other radii.

The spirals shown in Figure 6 have pattern speeds that approximately match the angular speed of the disk at the radii where they are most prominent. This is seen in the spectrograms of Figure 7, where we show the power spectra for the $m=4,6,7$, and 8 harmonic modes, measured from snapshots sampled every $5 \mathrm{Myr}$ for the spiral disk in the $930<t / \mathrm{Myr}<1260$ interval. Note that each mode spans a limited radial range and that the higher modes travel with a pattern speed similar to that of circular orbits at that radius.

As discussed by D'Onghia et al. (2013), arms in the pattern fade away and recur while traveling at about the circular angular frequency, in a seemingly "selfperpetuating" cycle. This implies that stars on nearly circular orbits will be consistently near CR of the spiral mode that dominates at each radius, favoring the corotation scattering mechanism of Sellwood \& Binney (2002). We examine its effect over many rotation periods next.

\section{RESULTS}

\subsection{Radial Migration}

In a stationary potential and in the absence of nonaxisymmetric perturbations disk stars should conserve both their energy, $E$, and the $z$-component of their specific angular momentum, $J_{z}$. The presence of the transient spiral patterns discussed in the last section, however, leads to exchanges of angular momentum amongst disk particles and to variations in their orbital energies.

We examine these changes by selecting, at $t_{0}$, particles within two $500 \mathrm{pc}$ wide cylindrical shells centered at $R=5$ and $8 \mathrm{kpc}$, respectively. We track these particles at later times and compare their current energies and

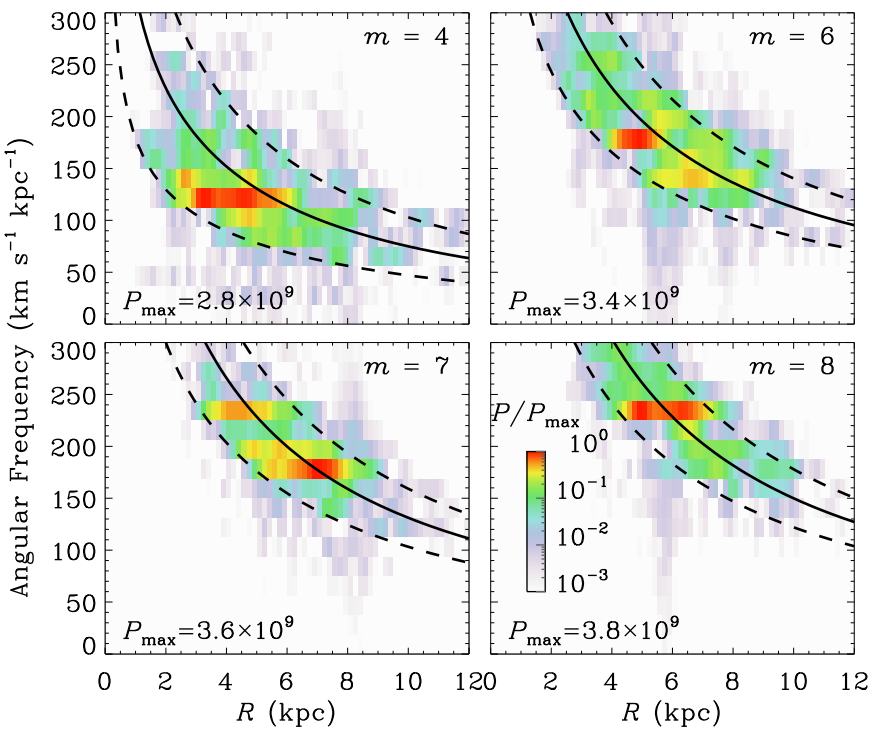

Figure 7. Power as a function of radius and frequency for different Fourier harmonics, $m$, in the spiral disk. The spectrogram is computed using snapshots spaced by $5 \mathrm{Myr}$ in the time interval $930<t / \mathrm{Myr}<1260$. In each panel, the solid line shows $m$ times the circular angular frequency, $\Omega_{\text {circ }}=V_{\text {circ }} / R$. The dashed lines correspond to the Lindblad resonances, $m \Omega_{\text {circ }} \pm \kappa$, where $\kappa$ is the usual epicycle frequency. Note the presence of persistent multi-arm wave-like perturbations that travel at roughly the circular orbit speed at the radius where they dominate.

angular momenta with those at $t_{0}$. The comparison is shown in Figure 8 for the control simulation (at $\left.t_{1}\right)$ and for the spiral simulation at $t_{1}, t_{2}$ and $t_{3}$.

Particles in the control simulation exhibit very slight changes in their values of $E$ and $J_{z}$, as expected given the smooth radial and azimuthal structure of the control 


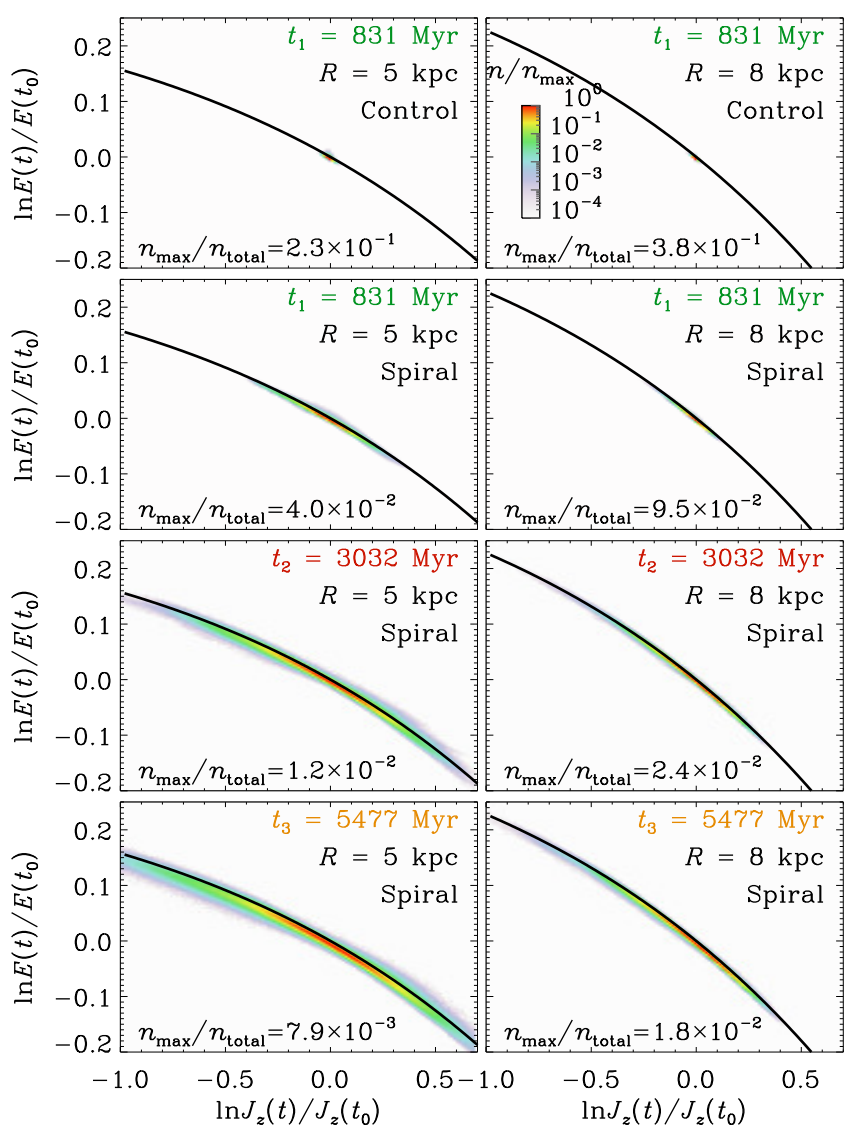

Figure 8. Changes in energy, $E$, and of orbital angular momentum $J_{z}$ measured for particles identified at $t_{0}$ at two different radii: $R=5 \pm 0.25 \mathrm{kpc}$ (left) and $R=8 \pm 0.25 \mathrm{kpc}$ (right). The top row show the control simulation; the rest correspond to the spiral disk, at the times labeled in each panel. The solid line shows the changes in $E$ and $J_{z}$ needed for a particles to remain on a circular orbit. Stars are binned in a $128 \times 128$ grid, with bin sizes of $\delta \ln E=3.5 \times 10^{-3}$ and $\delta \ln J_{z}=1.3 \times 10^{-2}$. The color scale encodes the number of points in each pixel $n$ normalized by the maximum $n_{\max }$.

disk at all times during its evolution.

Particles in the spiral simulation, on the other hand, undergo correlated changes in $E$ and $J_{z}$ that can reach (and exceed, in some cases) a factor of $\sim 2$ in $J_{z}$. Recalling that the guiding center radius, $R_{\mathrm{g}}$, of a star in a nearly circular orbit is directly proportional to $J_{z}$;

$$
J_{z}\left(R_{\mathrm{g}}\right)=R_{\mathrm{g}} V_{\text {circ }}\left(R_{\mathrm{g}}\right),
$$

(where $V_{\text {circ }}^{2}(R)=R \partial \Phi / \partial R$ and $\Phi$ is the gravitational potential on the disk plane) this implies changes in the guiding center that can exceed a factor of $\sim 2$.

These variations, however, do not alter the nearly circular nature of the orbits. Indeed, the correlated changes in $E$ and $J_{z}$ keep the stars very close to the minimum energy consistent with its angular momentum (indicated by the solid curves in Figure 8), which defines circular orbits. Thus, the spiral perturbations shown in Figs. 2 and 6 cause substantial migration in the spiral disk with minimal heating, as envisioned in the CR mechanism of Sellwood \& Binney (2002).

Figure 8 also hints that, as time goes by, the radial displacements of disk particles increase monotonically. We

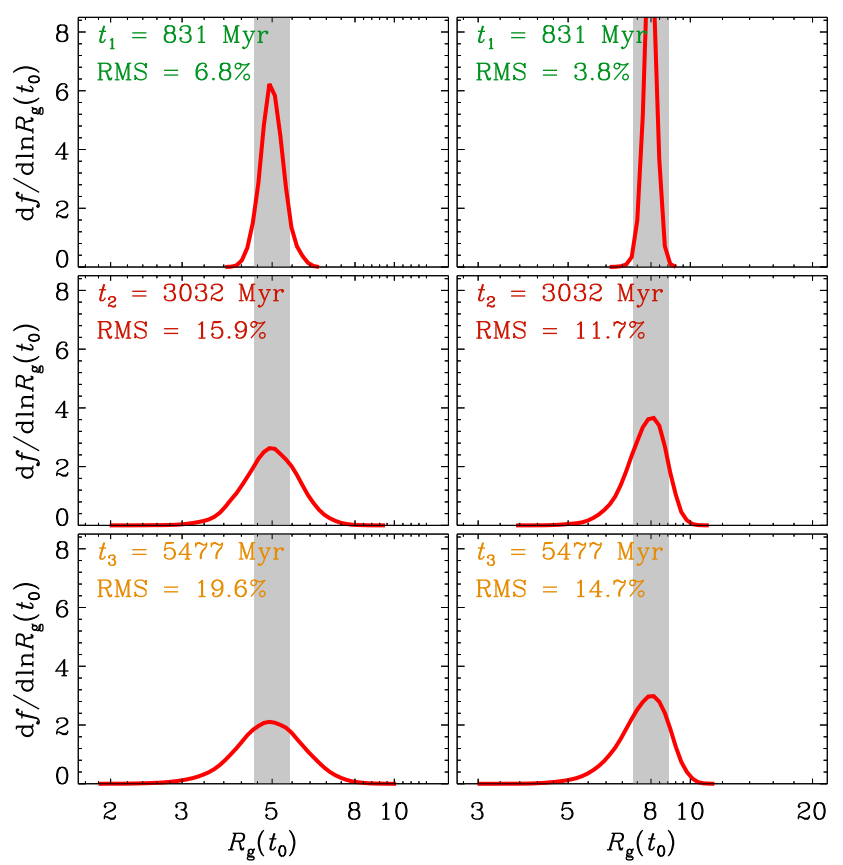

Figure 9. Distribution, at $t_{0}$, of guiding center radii of particles that migrate, by $t_{1}$ (top), $t_{2}$ (middle) and $t_{3}$ (bottom), to $R_{g}=$ $5 \pm 0.5 \mathrm{kpc}$ (left column) or $R_{g}=8 \pm 0.8 \mathrm{kpc}$. Labels in each panel indicate the rms of the distribution, which increases roughly like $t^{1 / 2}$.

show this more explicitly in Figure 9, where we plot the distribution of guiding center radii at $t=t_{0}$ of particles that migrate at later times to $R_{\mathrm{g}}(t)=5 \pm 0.5 \mathrm{kpc}$ (left panels) or $8 \pm 0.8 \mathrm{kpc}$ (right panels). The width of these distributions increase with time roughly as $t^{1 / 2}$, suggestive of a simple diffusion mechanism.

After more than 5 Gyr of evolution (i.e., at $t_{3}$ ) more than $10 \%$ of particles with $R_{\mathrm{g}}=5 \pm 0.5 \mathrm{kpc}$ have migrated to their current orbits from radii that were initially either $40 \%$ smaller or larger. At $8 \mathrm{kpc}$ the fraction of such extreme migrators reduces to $6 \%$ in the same time, presumably because of the longer orbital times characteristic of larger radii. The radial distribution of migrators at $8 \mathrm{kpc}$ is also clearly asymmetric, with a larger fraction of extreme migrators that come from the inner regions. This is again not surprising, since there are few particles beyond $8 \mathrm{kpc}$ in the disk.

We show in Figure 10 the evolution in radius of a few "extreme" migrators that have moved roughly 50\% inward or outward to reach their final radius. The top left and right panels correspond to migrators that reach $R_{\mathrm{g}} \sim 5$ or $8 \mathrm{kpc}$ at $t_{3}$, respectively. In spite of the large changes in guiding center, the migrators' orbital circularities barely change. This is shown in the bottom panels of Figure 10, where we plot the evolution of the circularity parameter, $\epsilon_{J}=J_{z} / J_{\text {circ }}(E)$, defined as the specific angular momentum in units of that of a circular orbit of the same energy. As these panels show, even extreme migrators stay on nearly circular orbits throughout the evolution.

\subsection{Migrator Properties}




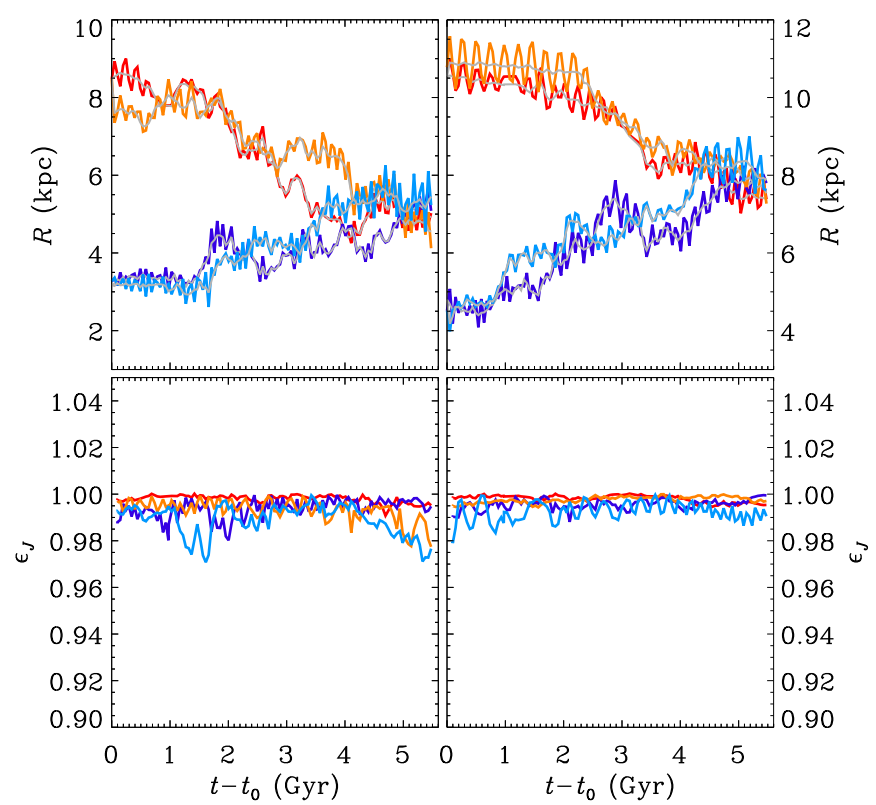

Figure 10. Top: orbits of extreme migrators that end up at $R \approx 5$ kpc (left panel) and at $R=8 \mathrm{kpc}$ (right panel). Two outward and two inward migrators are shown in each panel. In each case the gray line shows the instantaneous guiding center of the orbit. The bottom panels show the evolution of the circularity parameter, $\epsilon_{J}=J_{z} / J_{\text {circ }}(E)$, of the orbit of each migrator shown in the top panels. Note that the orbits of the migrators remain nearly circular despite the large changes in orbital angular momentum.

We explore the properties of migrators in Figure 11, where we show, in the left-hand panels, the vertical velocity dispersion of particles with guiding center radii $R_{\mathrm{g}}(t)=5 \pm 0.5 \mathrm{kpc}$ (shaded area), as a function of their original guiding center, $R_{\mathrm{g}}\left(t_{0}\right)$. Particles to the left of the shaded area have migrated outwards to reach $\sim 5 \mathrm{kpc}$; those to the right have migrated inward. For reference, the thick dashed curves in each panel of this figure indicate the vertical velocity dispersion profile of all particles in the disk, $\sigma_{z}(R)$.

The blue curves in Figure 11 show the current velocity dispersion of subsets of particles binned according to $R_{\mathrm{g}}\left(t_{0}\right)$. The inverted "U" shape indicates that migrators are a kinematically biased population with lower velocity dispersions than non-migrators, a bias that increases the farther away a particle has migrated from in order to reach its current radius. The bias affects both inward and outward migrators, and holds at all times. The bias is not only kinematical; as expected given their low vertical velocity dispersion, the vertical distribution of migrators is also thinner than that of non-migrators, as shown by their current vertical scaleheight (see the blue curves in the right-hand panels of Figure 11). Again, the farther particles have migrated from the thinner their current vertical distribution.

The cause of this vertical bias is twofold. Firstly, migrating stars are a heavily-biased subset of particles at their original radii, a "provenance bias" that increases the farther a particle migrates in a given time. This may be seen by comparing the red curves in Figure 11, which show the velocity dispersion and scaleheight of migrators at their initial radius, with the dashed black line, which shows the same but averaged for all stars at that radius.
The same vertical bias is clearly present at their radius of origin: radial migration mainly affects thinner-thanaverage populations at both their destination and provenance radii.

A second reason for the migrators' vertical bias may be seen by comparing the initial and final velocity dispersions of migrating stars. This is given by the difference, at given $R_{\mathrm{g}}\left(t_{0}\right)$, between the red and blue curves in Figure 11. Outward migrators (to the left of the gray zone) clearly cool as they move out (i.e., the blue curve is below the red), whereas the opposite holds for inward migrators. We note that this in itself would not be enough to explain the vertical bias at the final radius, since outward (inward) migrators originate in regions of higher (lower) velocity dispersion of the disk. However, Figure 11 shows that such cooling or heating is but a small correction to the "provenance bias" affecting all migrators.

We have checked that a similar provenance bias and heating/cooling process affect migrators at other radii, and that similar results are obtained when analyzing the other two components of the velocity dispersion, $\sigma_{R}$ and $\sigma_{\phi}$. Therefore, radial migration does not only operate most effectively on particles closest to the disk, but also on those closest to circular orbits. These biases have been hinted at in earlier work (see, e.g., Minchev et al. 2012b; Solway et al. 2012; Bird et al. 2012; Roškar et al. 2013) but their importance has not been properly appreciated, obscured perhaps by the concurrence of other heating mechanisms (i.e., satellite perturbations, disk growth, major disk instabilities) whose effectiveness in displacing stars radially can exceed that of the corotation scattering mechanism we focus on here (although see the case of the low surface brightness disk galaxy model explored in Loebman et al. 2011).

With the benefit of hindsight, our results are not surprising. Radial migration is caused by non-axisymmetric patterns in the disk that are more readily felt by stars that remain closer to the disk during their orbits; thus, the smaller the vertical amplitude of their oscillations the farther stars can migrate. This is, of course, true at all radii affected by the spiral patterns. If radial migration is not to cause vertical heating or a change in the surface density profile (as is the case in our simulation) then migrating stars at their final location must simply "substitute" other stars that have migrated off, which were themselves biased in the same way. In other words, to first order radial migration leads stars to merely "exchange" places in the disk, and affects first and foremost stars in the very thin disk.

\subsection{Effects of Radial Migration}

Our results extend and clarify earlier work on the possible effects of radial migration. For example, some authors have argued that outward migration causes the disk to thicken because it would shift stars with high vertical kinetic energy to regions where the disk gravity is lessened, an mechanism that Schönrich \& Binney (2009) have cited as a plausible origin for the Galactic thick disk. This assumption has been qualified and contested in later work, which have argued that migrating stars should cool vertically if they migrate out and heat up if they migrate inward. This was first proposed by Minchev et al. (2012a), who argued that such kinematic changes might signal the conservation of the vertical action, a 


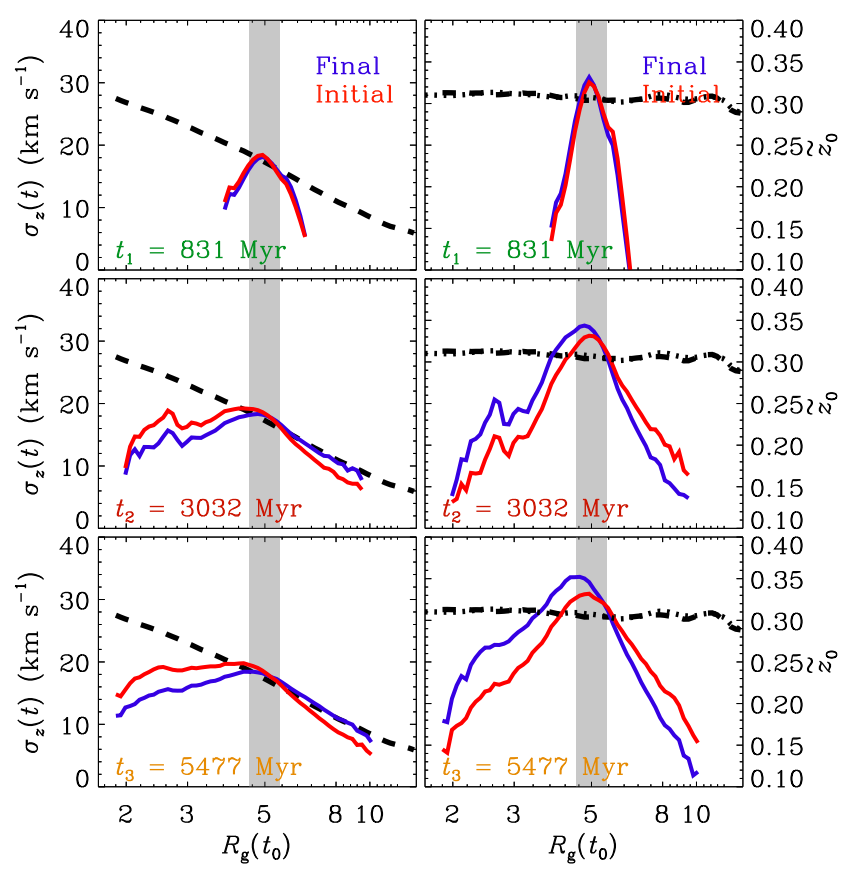

Figure 11. Left column: vertical velocity dispersion of particles that migrate to $R_{\mathrm{g}}\left(t_{3}\right)=5 \pm 0.5 \mathrm{kpc}$, shown as a function of their initial guiding center radius, $R_{\mathrm{g}}\left(t_{0}\right)$. The velocity dispersion profile of the whole disk is shown by the dashed black lines. Blue corresponds to velocity dispersions at $t_{3}$; red indicates $\sigma_{z}$ at $t_{0}$. Right column: same as left panels, but for the scale-height, defined as $\widetilde{z}_{0}=(2 \sqrt{3} / \pi)\left\langle z^{2}\right\rangle^{1 / 2}$, and normalized so that $\widetilde{z}_{0}=z_{0}$ for a vertical profile of the form $\zeta(z)=1 / 2 \operatorname{sech}^{2} z / z_{0}$ (see Eq. (1)).

result that has been further elaborated in more recent work (Solway et al. 2012; Minchev et al. 2012b; Roškar et al. 2013).

Our results help clarify this issue. Although it is true that migrators heat or cool as they move in or out of the disk, these changes play only a small role in determining the final vertical distribution of migrators. This is mainly set by the provenance bias we describe above, which has not been fully accounted for in earlier work. Because the bias adds to the cooling associated with outward migration, our results suggest that "hick disks" might not result from the only effect of radial migration, at least in multi-armed spiral galaxies.

Of course, this does not rule out the possibility that thick disk-stars in the solar neighborhood might have formed elsewhere in the Galaxy: an external origin (Abadi et al. 2003) or a pre-existing thin disk heated by external perturbations (Quinn et al. 1993), early mergers (Brook et al. 2004), or the deepening potential of the forming Galaxy, remain viable options for the origin of the Galactic thick disk.

Our results also suggest that radial migration could have had a substantial effect on the thin disk and that, in local volumes such as the solar neighborhood, the most extreme migrators might actually be found amongst stars closest to the disk. This is consistent with observations that suggest that some of the clearest signs of migration are found amongst thin disk stars.

One compelling example involves the origin of the large spread in metallicity of the thin disk, which spans the range $-0.7<[\mathrm{Fe} / \mathrm{H}]<0.3$ (see, e.g., Navarro et al. 2011, and references therein). If the spread is caused by radial migration of stars from the inner and outer Galaxy, then one would expect that those most chemically distinct should correspond, on average, to the most extreme migrators.

As discussed, for example, by Haywood (2008), this might explain the puzzling anti-correlation between metallicity and rotation velocity found for stars in the thin disk. In this scenario, the anti-correlation merely reflects the difference in angular momentum expected for inward and outward migrators that have reached the solar neighborhood. Arrivals from the outer disk (presumably those populating the metal-poor tail of the thin disk) should have higher-than-average angular momentum, while the opposite should hold for those coming from the inner disk. The net effect is a negative gradient in the $V_{\phi}$ versus $[\mathrm{Fe} / \mathrm{H}]$ relation (or, equivalently, in $R_{\mathrm{g}}$ versus $[\mathrm{Fe} / \mathrm{H}]$ ), which is indeed observed (Lee et al. 2011; Haywood et al. 2013; Recio-Blanco et al. 2014).

Our results confirm this expectation. Figure 12 shows, for particles identified in a narrow cylindrical shell of radius $R=5 \pm 0.5 \mathrm{kpc}$ at the final time, $t_{3}$, the azimuthal velocity, $V_{\phi}\left(t_{3}\right)$, as a function of the initial guiding center radius, $R_{\mathrm{g}}\left(t_{0}\right)$. This figure shows that stars that have migrated inward from large radii tend to have larger $V_{\phi}$ (or, equivalently, larger $R_{\mathrm{g}}$ ) than those that have migrated outward from well within the inner disk, leading to a well defined correlation between rotation velocity and provenance radius. If radius correlates with metallicity, then a negative metallicity gradient in the Galaxy would give rise to an anti-correlation between $V_{\phi}$ and metal content such as observed.

On the other hand, we note that stars chemically identified to be part of the Galactic thick disk by their $\alpha$ enhancement (see, e.g., Navarro et al. 2011) show a gradient in the $V_{\phi}$ versus $[\mathrm{Fe} / \mathrm{H}]$ relation of opposite sign (see, e.g., Lee et al. 2011; Kordopatis et al. 2013; RecioBlanco et al. 2014), a result that is difficult to reconcile in our models assuming a migration-driven scenario for the origin of the thick disk. However we notice that in this scenario the anti-correlation has been reproduced as reported in Figure 15 of Loebman et al. (2011). The reason of the differences between our results and previous results might be in the different models of galaxies adopted and will be investigated in a forthcoming work. Note that the different signs of the gradient suggest a qualitative distinction in the origin of $\alpha$-poor (thin-disk) stars and $\alpha$ rich (thick-disk) stars. Such kinematic distinction might also present an interesting challenge to models that view the Galactic disk as a single entity made by the overlap of several mono-abundance populations (Bovy et al. 2012a,b).

Finally, our results suggest an extra consistency check of the migration interpretation of the thin disk $V_{\phi}$ versus $[\mathrm{Fe} / \mathrm{H}]$ anti-correlation: those that migrate the most should also have lower-than-average vertical velocity dispersions. In this interpretation, both unusually metalpoor or unusually metal-rich stars should have lower scaleheights, although the trend might be more readily noticeable in the metal-poor population (see Figure 11). No such effect has been reported in the literature, but this may be due to difficulties in separating thin-disk from thick-disk stars at the metal-poor end. Indeed, we note that a kindred effect has been argued by Minchev 


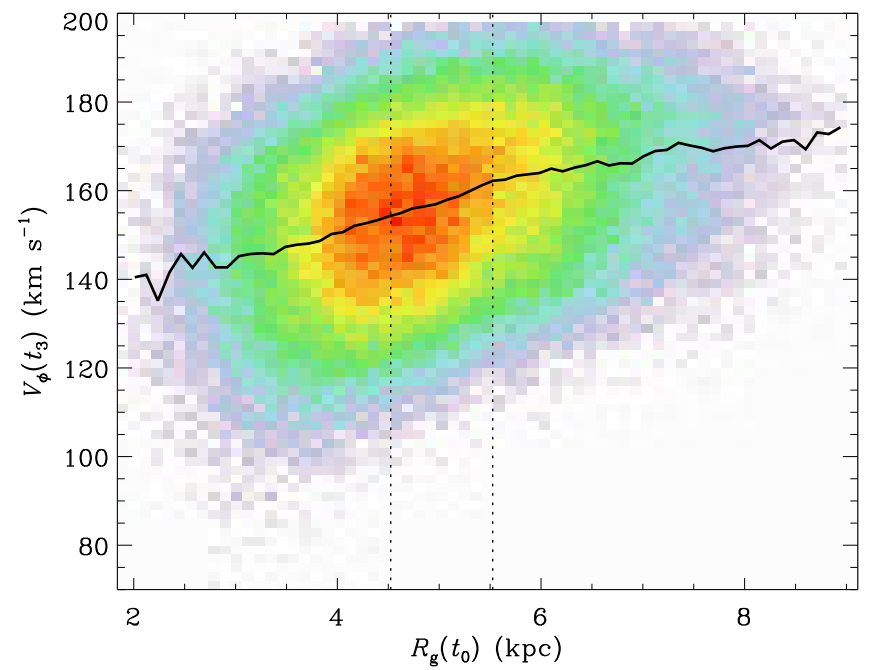

Figure 12. Azimuthal velocity versus initial guiding center radius for particles identified, at the final time $t_{3}$, within a cylindrical shell of radius $R=5 \pm 0.5 \mathrm{kpc}$. All of these stars have, at $t_{3}$, guiding center radii in the range $3.3<R_{\mathrm{g}} / \mathrm{kpc}<6.2$. Note that inward migrators from the outer disk have higher rotation velocities than outward migrators from the inner galaxy.

et al. (2014) to explain the drop in $\sigma_{z}$ observed for the most $\alpha$-rich stars in their sample.

\section{SUMMARY AND CONCLUSIONS}

We have analyzed the effects of a persistent, multiarm spiral pattern on the radial distribution of particles in an exponential thin disk. Our disk is modeled with $10^{8}$ self-gravitating particles and a rigid halo potential, thus minimizing the effects of noise. Evolved in isolation, the disk develops no obvious non-axisymmetric patterns nor is subject to the violent instabilities that have often plagued $N$-body models of cold stellar disks.

Spiral patterns are seeded in the disk by the temporary addition of massive perturbers intended to simulate the presence of molecular clouds. The disk responds to the molecular clouds by developing an intricate multi-arm spiral pattern that persists, after the perturbers are removed, for over 5 Gyr. Interestingly, the perturbations cause only minor heating and have no discernible effect on the vertical structure of the disk. Thus, our simulations are especially well suited to analyze the effect of long-lived spiral patterns on the orbits of disk stars.

Stars in the disk respond to the spiral perturbations by migrating widely through the disk whilst largely preserving the nearly circular nature of their orbits. Adopting this as a definition of radial migration, we focus our analysis on the properties of migrators and on the effects of radial migration on the vertical structure of the disk. Our main conclusions may be summarized as follows.

- Radial migration affects a heavily biased subset of disk particles, mostly those with small vertical oscillations and close to circular orbits. This implies a "provenance bias" where migrators sample a very select population of stars at their radius of origin. The more a particle migrates the stronger the bias, implying that the most extreme migrators should be found, at given radius, amongst the stars whose orbits deviate the least from the local standard of rest.

- Migration affects systematically the kinematics of stars; outward migrators cool down whereas inward migrators heat up. The magnitude of this effect is, however, small compared to the provenance bias referred to above. Indeed, although inward migrators heat up as they move in, they come from regions that are cooler than their destination and, in addition, they constitute a cooler subset of particles from that region. The small amount of heating they experience as they move in is not enough to revert the bias. In general, migrators have, at given radius, much smaller scaleheights and velocity dispersion than non-migrators.

- Migrators do not "accommodate" to match the properties of their new surroundings. Rather, they replace particles that were originally resident there and that have migrated off. The latter are predominantly stars on very nearly circular orbits, and so are the new arrivals.

- Radial migration might play a substantial role in determining the local properties of the thin disk of the Galaxy. In agreement with earlier suggestions, our results suggest that radial migration might help to explain the large spread in metallicity observed for stars in the ( $\alpha$-poor) thin disk as well as the anti-correlation observed between metallicity and rotation velocity of thin-disk stars.

Our results endorse the view that migration might play a substantial role in the radial redistribution of stars in galaxies that have experienced sustained spiral structure. Although we have tried to emphasize the results that seem of general applicability, we also recognize that our conclusions are based on a single simulation that, in many ways, fails to capture the fascinating complexity of galaxies like our own Milky Way. The evolving potential of the forming galaxy; the accretion of gas and the ongoing formation of stars; the effects of satellites and substructure; the presence of a bar and of evolving spirals; all must have left an imprint on the structure of the Galaxy that our simulations cannot attempt to match.

Further, our simulation adopts a disk that is lighter than that of the Milky Way and whose spiral patterns, therefore, might differ from that of the Galaxy (see also Loebman et al. 2011). We cannot therefore rule out that other disturbances may give rise to a thick disk, as argued by Roškar et al. (2013). A more massive disk would probably sport spirals with fewer arms that might lead to faster heating. Although we do not believe that this would affect qualitatively our conclusions, a more definitive, quantitative comparison with observation should be attempted with models that reproduce better the known structural parameters of the Galaxy. Still, we believe that simplified models like the one we discuss here are useful, since they allow us to isolate and understand the effects of radial migration so as to inform the analysis of more ambitious modeling.

This work is funded by NSF grant No. 1211258 and 
NASA grant No. 13-ATP13-0053. ED gratefully acknowledges the support of the Alfred P. Sloan Foundation. CV-C, ED and JN express their appreciation towards the Aspen Center for Physics for their hospitality. MA and JN acknowledge the support of ANPCyT, Argentina by grant No. PICT 2012-1137. This work was supported in part by the National Science Foundation under grant No. PHYS-1066293. This simulation has been run on Odyssey at Harvard and on the High Performance Computing cluster provided by the Advanced Computing Infrastructure (ACI) and Center for High Throughput Computing (CHTC) at the University of Wisconsin. This work was supported in part by the National Science Foundation under Grant No. PHYS-1066293.

\section{REFERENCES}

Abadi, M. G., Navarro, J. F., Steinmetz, M., \& Eke, V. R. 2003, ApJ, 597, 21

Bergemann, M., Ruchti, G. R., Serenelli, A., et al. 2014, A\&A, 565, A 89

Bird, J. C., Kazantzidis, S., \& Weinberg, D. H. 2012, MNRAS, 420, 913

Bovy, J., Rix, H.-W., \& Hogg, D. W. 2012a, ApJ, 751, 131

Bovy, J., Rix, H.-W., Liu, C., et al. 2012b, ApJ, 753, 148

Brook, C. B., Kawata, D., Gibson, B. K., \& Freeman, K. C. 2004, ApJ, 612, 894

Brunetti, M., Chiappini, C., \& Pfenniger, D. 2011, A\&A, 534, A75

D'Onghia, E., Vogelsberger, M., \& Hernquist, L. 2013, ApJ, 766, 34

Edvardsson, B., Andersen, J., Gustafsson, B., et al. 1993, A\&A, 275,101

Feltzing, S., \& Chiba, M. 2013, New Astronomy Review, 57, 80
Grand, R. J. J., Kawata, D., \& Cropper, M. 2012, MNRAS, 421, 1529

Haywood, M. 2008, MNRAS, 388, 1175

Haywood, M., Di Matteo, P., Lehnert, M. D., Katz, D., \& Gómez, A. 2013, A\&A, 560, A109

Hernquist, L. 1990, ApJ, 356, 359

-. 1993, ApJS, 86, 389

Kazantzidis, S., Bullock, J. S., Zentner, A. R., Kravtsov, A. V., \& Moustakas, L. A. 2008, ApJ, 688, 254

Kordopatis, G., Gilmore, G., Wyse, R. F. G., et al. 2013, MNRAS, 436, 3231

Lee, Y. S., Beers, T. C., An, D., et al. 2011, ApJ, 738, 187

Loebman, S. R., Roškar, R., Debattista, V. P., et al. 2011, ApJ, 737,8

Minchev, I., \& Famaey, B. 2010, ApJ, 722, 112

Minchev, I., Famaey, B., Combes, F., et al. 2011, A\&A, 527, A147

Minchev, I., Famaey, B., Quillen, A. C., \& Dehnen, W. 2012a, in European Physical Journal Web of Conferences, Vol. 19, European Physical Journal Web of Conferences, 7002

Minchev, I., Famaey, B., Quillen, A. C., et al. 2012b, A\&A, 548, A127

Minchev, I., Chiappini, C., Martig, M., et al. 2014, ApJ, 781, L20

Navarro, J. F., Abadi, M. G., Venn, K. A., Freeman, K. C., \& Anguiano, B. 2011, MNRAS, 412, 1203

Quinn, P. J., Hernquist, L., \& Fullagar, D. P. 1993, ApJ, 403, 74

Recio-Blanco, A., de Laverny, P., Kordopatis, G., et al. 2014, A\&A, 567, A5

Roškar, R., Debattista, V. P., \& Loebman, S. R. 2013, MNRAS, 433, 976

Roškar, R., Debattista, V. P., Quinn, T. R., Stinson, G. S., \& Wadsley, J. 2008, ApJ, 684, L79

Roškar, R., Debattista, V. P., Quinn, T. R., \& Wadsley, J. 2012, MNRAS, 426, 2089

Schönrich, R.. \& Binney, J. 2009, MNRAS, 396, 203

Sellwood, J. A., \& Binney, J. J. 2002, MNRAS, 336, 785

Sellwood, J. A., \& Carlberg, R. G. 2014, ApJ, 785, 137

Solway, M., Sellwood, J. A., \& Schönrich, R. 2012, MNRAS, 422, 1363

Springel, V. 2005, MNRAS, 364, 1105

Springel, V., Di Matteo, T., \& Hernquist, L. 2005, MNRAS, 361, 776

Villalobos, Á., \& Helmi, A. 2008, MNRAS, 391, 1806

Wielen, R., Fuchs, B., \& Dettbarn, C. 1996, A\&A, 314, 438 